\title{
Téoros
}

Revue de recherche en tourisme

\section{Voyage terminologique au pays des congrès}

\section{Charles Dupont}

Volume 4, numéro 3, novembre 1985

L'essor de l'industrie des congrès

URI : https://id.erudit.org/iderudit/1080785ar

DOI : https://doi.org/10.7202/1080785ar

Aller au sommaire du numéro

Éditeur(s)

Université du Québec à Montréal

ISSN

0712-8657 (imprimé)

1923-2705 (numérique)

Découvrir la revue

Citer cet article

Dupont, C. (1985). Voyage terminologique au pays des congrès. Téoros, 4(3), 4-5. https://doi.org/10.7202/1080785ar d'utilisation que vous pouvez consulter en ligne.

https://apropos.erudit.org/fr/usagers/politique-dutilisation/ 


\title{
Voyage terminologique au pays des congrès
}

\author{
par Charles Dupont
}

Le présent texte examine la terminologie relative à l'organisation et à la tenue d'un congrẻs. Chaque notion clé est identifiée par un terme en caractère gras suivi de son équivalent anglais entre guillemets.

Cette présentation est non seulement nécessaire due au caractère bilingue de notre environnement, mais aussi parce que le phénomène "congrès" est largement etatsunien. Nous nous excusons de I'emploi répété du mot "congrès" qui constitue aux fins de l'article un générique pour tous les types de réunion.

\section{Les acteurs}

L'initiative de tenir un congrès sconventions est le plus souvent prise par une association représentant des intéréts professionnels ou sociaux. On pense, par exemple, au congrès de la Fédération internationale pharmaceutique ou à celui des Alcooliques Anonymes qui se sont tenus a Montréal en 1985. Outre évidemment le groupe intéressé a tenir un congrẻs, son organisation nécessite la participation de trois autres figurants: un office des congrès, un hôtel ou un palais des congrès et un organisateur de congrès. Voyons brièvement le röle de chacun.

L'Office des congrès uconvention bureaus, organisme public chargé de la promotion d'une ville, veille à ce que chaque association obtienne les meilleurs services compte tenue de la nature de l'événement et, par voie conséquence, à ce que la réputation de la ville soit préservée pour la tenue de congrès.

L'hôtel shotel's est depuis longtemps un foumisseur d'installations afacilitiess (salles ou salons afunction rooms pour tenir une conférence ou une exposition ou encore $y$ aménager une salle de pressel et de prestations uservicen (traitance $\alpha$ catering interprétation ainterpretations, etc.) pour la tenue de congrès; certains sont si

\footnotetext{
- Charlos Dupont est terminclogue principal au sein des services linguistiques d'Air Canada es est auteur du Vocabulaire du tourisme (Linguatech, 1979) et du Dictionnaire de I"hótellerie at de la restouration ISodilis, 1981). Les termes cites dans le texte foment un extrait d'un manuscrit non publie.
}

toumés vers ce marché, qu'on les désigne de l'expression hôtel de congrès kconvention hotelw. Depuis quelques années, nombre de villes désirant accueillir les congrès internationaux et les grandes expositions ont été dotées d'un palais des congrès cconvention centers dont la capacité d'accueil des installations dépasse celle d'un hötel. Quand un grand congrès se tient dans un tel complexe, cela nécessite l'organisation d'une centrale d'hébergement shousing bureaux qui coordonne la participation des hôtels oủ logeront les congressistes aconvention-goer; conventioneern. Enfin, les responsabilitếs relatives aux congrès dans ces établissements sont réparties entre le directeur des réservations areservation managern, le directeur des congrès uconvention services managers, le directeur du hall d'exposition sexhibit hall managers, le directeur de la restauration ufood and beverage managers et le directeur des banquets ebanquet managerb.

L'organisateur de congrès «meeting planner ou professional convention organizers est un nouveau protessionnel qui prend en charge la logistique d'un congrès pour le compte d'une association. II ne foumit pas d'installations ou de prestations comme telles, mais il met en place, en négociant des contrats avac des prestataires umeeting support suppliern, tous les éléments nécessaires à la tenue du congrès.

\section{Le processus décisionnel}

Le processus menant á la tenue d'un congrês s'échelonne sur une période pouvant varier de quelques mois à plusieurs années pour les grands congrès. La premięre décision porte le plus souvent sur le lieu du congrès uconvention sitew. II s'agit dans de nombreux cas d'une décision prise après étude des candidatures présentées par des sections achaptern de l'association.

L'association confie à un comité organisateur korganizing committees la responsabilité de préparer le congrès et établit avec ce comité un calendrier aschedule; timetablex des préparatifs. Crest, dans beaucoup de cas, ả ce moment que l'association retient les services d'un organisateur de congres pour completer l'état-major du congrès aconvention officialsy.

Les établissements en mesure d'accueillir le congrès aux dates retenues sont invités a soumettre une proposition aproposalp dans lacuelle ils précisent les prestations offertes et les tarifs. Suite à cela, l'organisateur de congrès choisit celle qui est la plus en harmonie avec la nature du congrès à tenir et fait, par le biais d'une lettre d'entente sletter of agreements, une réservation d'amorce utentative reservation». Cette réservation tient jusqu'à une date d'option woption daten, c'est-à-dire la date à laquelle on doit la confirmer ou I'annuler.

Le premier contrat négocié par l'organisateur de congrès avec le palais des congrès ou l'hotel porte sur les installations à four" nir, les manifestations afunctions à accueillir et les chambres à retenir. L'organisateur s'appuie sur les données des congrès antérieurs pour évaluer les besoins, mais cherche, au départ, à retenir le maximum d'installations et de chambres pour obtenir les meilleurs tarifs uratew. Ce contrat comprend, toutefois, une (parfois plusieurs) date de confirmation acut-off dates qui oblige I'organisateur, $x$ jours avant l'ouverture du congrès, à s'engager à payer pour les installations et les chambres retenues ou à en remettre une partie. Le même processus se déroule pour les réceptions $\alpha 1$ - food function (langage technique) 2 -reception (langue générale)s pour lesquelles il doit préciser le nombre de couverts acoverw bien qu'ici il conserve une marge deeway.

\section{L'organisation}

L'organisation d'un congrès, comme il a été mentionné plus tôt, est un long processus nécessitant la préparation d'un scéna= rio *convention resumes des manifestations (nature, horaire, etc.) et pour chacune des manifestations, un plan d'allocation ufunction sheets précise l'aménagement désiré, l'équipement nécessaire et même le plan de table wseating lists s'il s'agit d'une réception. Le scénario et le plan d'allocation sont des documents dont le contenu se précise à mesure qu'approche l'événement. 
Pour assurer le success et le bon déroulement du congrès, l'organisateur doit penser en fonction de trois groupes: les participants, les conférenciers et la presse.

La tenue d'un congrès doit être abondamment annoncée si on veut attirer le maximum de participants eparticipant: attendeen. Aussi, on fait parvenir aux membres de l'association une brochure upamphletw annoncant le thème uthemen et les dates du congrès, le programme préliminaire "preliminary program» et les frais (droits) d'inscription uregistration feen. Ce document renferme un bulletin d'inscription aregistration form» sur lequel les intéressés pourront mentionner le type d'hébergement saccommodation typen désiré et les activités sociales usocial functionm auxquelles ils comptent participer. L'envoi comprend aussi de la documentation touristique wsightseeing literatures sur les attractions wattractions, les tours de ville ou visites touristiques $\alpha$ sightseeing tournet les circuits pré- ou post-congrès apre- or post-convention tourn offerts.

Au moment de l'inscription uregistrations, le personnel a défả en main la liste des participants alist of participantsw inscrits et il est prêt â remettre les documents (badge ename card; badge", coupon-repas "meal ticket; meal vouchern et bons wvouchern pour les activités, programme uprograms, etc.) dont aura besoin le congressiste.

Les conférenciers «speaker» forment un groupe important dans un congrès, car leur nom, sans mentionner leurs idées, peut attirer des participants. Une attention particulière doit être accordée au premier conférencier akevnote speakers qui prononce le discours d'ouverture kkeynote speechw et au conférencier invité aguest speakers qui prend la parole à l'occasion d'une réception, puisque souvent ces gens donnent le ton à l'événement. Les conférenciers doivent recevoir des instructions précises afin que leur communication ou exposé «paperz demeure pertinent par rapport au thème du congrès et, surtout, ne dépasse pas le temps accordé dans le programme. Parfois, pour éviter qu'il y ait trop d'exposés mineurs, on offre la possibilité d'une présentation par affichage uposter presentationn.

Dans les grands congrès où il y a plusieurs langues de travail «working languagen, il faut retenir les services d'interprètes kinterpretery et fournir des écouteurs kreceivern aux participants. Cela permet au conférencier de s'exprimer dans un des langues de travail du congrés, alors que de la cabine d'interprétation ainterpreter roomy on transmet une traduction dans toutes les autres langues.

Enfin, la presse upressu. Si le congrès a des objectifs précis en terme d'impact professionnel, social ou commercial, il faut en informer le public en tenant des conféren- ces de presse upress conference» ou en émettant des communiqués anews releasey aux agences de presse unews agencym. Les relations avec la presse sont confiées dans les grands congrès à un attaché de presse «press officern.

La présence de journalistes nécessite la distribution d'accréditations de presse smedia credentialy et la remise d'un cahier de presse kpress kith donnant les grandes lignes du congrès, des informations sur l'association, les conférenciers, etc. Cette présence requiert aussi l'aménagement d'une salle de presse anewsroom; press roomy pour mener des entrevues et d'autres travaux professionnels.

Comme dernier point technique sur la prếparation d'un congrès, nous abordons les plans d'aménagement "floor plan» des salles. Les Étatsuniens sont passés maîtres des différentes façons de disposer chaises et tables et de leur attribuer un nom évocateur. Cet aspect de la préparation d'un congrès ne doit pas être négligé, car le confort des participants et leur degré d'attention aux exposés en dépendent; aussi, l'écartement wspacen entre les chaises et le pas «distance» entre les rangées doivent être soigneusement calculés.

Les aménagements les plus souvent retenus sont, pour les grandes salles; l'aménagement en amphithéatre $\mathrm{s}$ theater style setupw, en salle de classe aclassroom style setup» et en hémicycle usenate style setupi. Pour les groupes restreints, les types d'aménagement les plus courants sont l'aménagement en cadre ahollow square setup\%, l'aménagement en anneau ahollow circular setup " la table en fer à cheval whorseshoe setupw, la table en $\mathbf{E}$. $T$ ou $U$ \&E-shape, $\mathrm{T}$-shape or $\mathrm{U}$-shape setup $w_{s}$ et la table rectangulaire wboard of directors setupn.

Lorsque le congrès comporte aussi une exposition sexhibitions ou un salon wtrade shown, une organisation plus vaste doit être mise en place. II faut réserver un hall d'exposition wexhibition halls, préparer un manuel de l'exposant wexhibitor manuals, répondre aux demandes de stands kapplication for spacew, prévoir les temps de montage umove in et de démontage umove out», veiller à la répartition des stands cbooth assignments et à leur aménagement, déterminer si l'entrée est libre uopen admissions ou payante ucharge admissions, etc.

Pour ce qui concerne les stands $\alpha$ booth, ou kiosques comme on dit au Québec, leur présentation est en fonction de l'aménagement global de l'exposition. Les types les plus courants sont: les stands à trois faça-

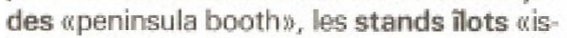
land boothn, les stands de ceinture aperimeter wall booths (ceux-ci encerclent le hall d'expositionl et les stands stan- dards \&standard booth» (les uns collés aux autres avec une seule façade).

\section{Le déroulement}

Pour terminer, faisons un court rappel des trois grands moments d'un congrès, c'està-dire les cérémonies d'ouverture wopening ceremoniesm, les travaux comme tels et les cérémonies de clôture kclosing ceremonies:

Patronnée par un président d'honneur "president of honours, la séance d'ouverture ou inaugurale wopening sessionx marque le coup d'envoi du congrès. Par la suite, les congressistes prennent part à des ateliers aworkshopw, participent à des séminaires useminary ou encore assistent à des conférences thèmes wtheme conferences et à des panels apanelw avant de se réunir périodiquement, ou à la fin du congrès, en séance plénière oplenary sessions. Cette séance plénière constitue souvent la séance de clóture uclosing session» où les premières conclusions du congrès sont avancées.

La dernière fonction officielle du comité organisateur est généralement la publication des actes aproceedingsw du congrès pour un compte rendu fidèle des conférences, exposés et débats. $f$

\section{Bibliographie sommaire \\ Sources francaises}

BAILLY, J.-C. Compte rendus du 1er séminairs international des directeurs et directeurs techniques des. centres de congrès et d'expositions - L'audio-visuel au service des palais des congrès. Genève: Institut international de recherche sur les congress, s.d. 36 feuillets.

BOUDON, Marcelle, Comment organiser rencontres et sessions. Paris: Le Centurion, 1974, 71 p

DUCHESNE, L.R. Manuel de l'organisateur de congrès. Bruxelles: Union des associations internationales, 1961. 98 p.

LANGUAF, Fi et al. Congrès, séminaires vovages de stimulation. Paris: PUF, 1900. Que sais-je? no 1855.

SPEECKAEAT, G.P. Les divers types de réunions internationales, I' aménagement des salles, l'équipement et les services. Bruxelles: Union des associations internationales, $1967.127 \mathrm{p}$.

Sources anolarses

ASTROFF, M.T. and Abbey, J.R. Convention Sales and Services. Dubuque (lowa): C. Brown Co. 1978, $448 \mathrm{p}$.

BERKMAN, Frank W. Convention Management and Service. East Lansing (Michigan): The Educational Institute of the Amorican Hotel and Motel Assoclation, 1978. $290 \mathrm{p}$.

Functional Room Set-Up Manual. New-York: Hotel Sales Association, $1969,44 \mathrm{p}$.

IAPCO. Glossary of Conference Terminology, Milan: Meeting and Congressi, 1982, $15 \mathrm{p}$.

Convention an American Institution. Cincinnati: International Association of Convention Bureaus, 1958. $138 \mathrm{p}$.

LAWSON, Fred Conference, Convention and Exhlbition Facilities. A Handbook of Planning, Design and Management, London: The Architectural Press, 1981. $268 \mathrm{p}$.

Convention Liaison Manuel. Washington: Conwention Liaison Council, 1980. $100 \mathrm{p}$ 